\title{
Design and Control of Unmanned Underwater Vehicle
}

\author{
Rehan Akbar ${ }^{1}$, Syed Raza Mehdi ${ }^{2}$, Aijaz Akhtar ${ }^{1}$, Bharat lal $^{1}$ Faizan $^{3}$, Nooruddin \\ Khaskheli ${ }^{4}$, Kamran Shahani ${ }^{2}$
}

\begin{abstract}
:
Unmanned Underwater Vehicles (UUV) are the robots that are used to monitor underwater environment at remote distance, either for inspection of underwater wreckage or for surveillance of underwater environment. Any such robot due to harshness of environment conditions needs a robust watertight design and requires various sensors for effective environment monitoring. The entire UUV structure is comprised of the vehicle, which is linked with operators on top of the surface by a set of cables that hold relevant cables for data and power communication. In this context, a lightweight underwater vehicle was designed using PVC (Polyvinyl Chloride) pipes and equipped with brushless DC motors, electronic speed controller, relay module, sonar, claw arm and Camera. It can navigate in six directions (up, down, left, right, forward, backward) and camera allows underwater surveillance of the environment, with sonar helping to indicate the target depth. The designed UUV can also perform basic pick and place task using a claw arm and is remotely operated using a joystick controller. Overall, the designed UUV prototype has shown its potential capabilities for underwater surveillance and environment monitoring through practical demonstrations.
\end{abstract}

Keywords: Automation; ROV; Sonar; joystick; manipulator;Arduino.

\section{Introduction}

The inventions of the UUV's were designed for underwater surveillance and to explore underwater life. it is widely used by navy for underwater inspection and it is also widely used by civilians in oil industries. Sometimes it's also known as underwater drones. Such vehicles can act as the eyes of undersea fleet. These are most recent demonstration of such vehicles, replacing divers to do work in the ocean. These vehicles, also known as Deep Submergence Vehicles (DSVs), were designed to go deeper than divers. These were configured for ocean exploration, science, rescue, and survey $[1,2]$. It is fundamentally a tethered submerged vehicle which consent the robots functioning to continue in comfy surroundings although it works in the perilous surroundings below. The entire UUV structure is linked with operators on the facade by a set of cables that lug electrical power, video and information signals reverse and forward among the

\footnotetext{
${ }^{1}$ Department of Electrical Engineering, Isra University Hyderabad, Pakistan

${ }^{2}$ Ocean optics and automation lab, Institute of ocean technology, Ocean College, Zhejiang University

Hangzhou 310058, China

${ }^{3}$ Institute of Marine Robotics, Ocean College, Zhejiang University;

${ }^{4}$ Institute of Coastal engineering, Ocean College, Zhejiang University;

Corresponding Author: kamranshahani@zju.edu.cn
} 
machinist and the vehicle. UUVs are relatively small, tethered, and unmanned vehicle that are able to work solely underwater within the handled and sustained ability [4]. Unmanned underwater vehicle varies in design modeling according to the desired operational depth and task. Mainly Autonomous Underwater Vehicles (AUVs) can be able to function near $200 \mathrm{~m}$ or consequently, with a little working ahead of $5000 \mathrm{~m}$. Autosub- 2 of the UK is usual of the intend of lots of AUVs. Long-range gliders (Simonetti, 1998) can also be considered as AUVs, although for the purposes of these review they are excluded because of the high power and payload-space requirements of current auditory instruments; gliders as well have limited straight actions which would build logical surveying challenges [5]. Due to the huge technical improvements obtained in past decade, it is probable to utilize robot's vehicle for undersea examination. In this approach, Remotely Operated Vehicles (ROVs) have been used carefully in the investigation of subsea phenomena as well as in the assemblage, examination and repair of offshore structures. Throughout the completion of a firm task with the robotic vehicle, the operator needs to observe and manage a number of parameter. If, for example, the adjustment to the position of ROV are made automatically, the precision will be greatly increased as compared to an observer controlling it manually [7-10]. This paper present the design of a UUV through an innovative display of thrusters with dynamical planned system in desire to be used in replication and manipulative of controllers. Finally, design and structure of prototype of the planned UUV, with brushless DC motors arrangement of the current work is presented. The compensation of using a proper arrangement of brushless DC motors is scheduled as under [3]. Reducing the number of required motors, at least six motors through propeller are essential to manage the UUV in its six degrees of freedom (DOF), while by brushless DC motors just three or utmost four DC brushless motors are essential in order to give the essential pressure. Materials Inventory of latest assembly being used in autonomous submerged robots will persistently increase. In addition, aluminum with carbon fiber can be utilized for such deep sea vehicles. In this prototype the PVC (Polyvinyl chloride) pipes are used in the assembly. These materials permit the UUV to be both lightweight and neutral buoyant, and to resist opposing friction. An example of underwater vehicle with similar materials is Deep glider, capable to submerge up to $6 \mathrm{~km}$ [4].

\section{Control System}

The control system designed for this UUV is simple and low cost. The vehicle is controlled on the front end by a PS2 joystick programmed with Arduino Nano as a master controller, which includes electronic components such as power regulators, live screen, battery bank and interfacing keys. Master controller via tether using serial communication (I2C) controls the slave controller. The designed assembly of UUV lying on simple design reduces drag, which improves the underwater manipulative performance of the vehicle. A proportional integration and derivation (PID) controller has been implemented effectively in this approach. The control system provides the controllable and desired thrust power to the designed vehicle. Different control methods of unmanned submerged vehicle are shown in Table I.

TABLE I. Control methods with restriction

\begin{tabular}{|c|l|}
\hline $\begin{array}{c}\text { Control } \\
\text { method }\end{array}$ & limitations \\
\hline PID & $\begin{array}{l}\text { Can't energetically recompense instead } \\
\text { un-modeled robot's hydraulics forces } \\
\text { or indefinite instability constraint design } \\
\text { opposing among response speed and } \\
\text { overshoot control }\end{array}$ \\
\hline $\begin{array}{c}\text { Descendi } \\
\text { ng form }\end{array}$ & $\begin{array}{l}\text { Might effortlessly escort to system } \\
\text { consequence manage precision }\end{array}$ \\
\hline Fuzzy & $\begin{array}{l}\text { Difficult to adjust the fuzzy system. } \\
\text { Overshoot prophecy instant not curved }\end{array}$ \\
\hline $\begin{array}{c}\text { Neural } \\
\text { Network }\end{array}$ & $\begin{array}{l}\text { Can't assemble the necessity of quick } \\
\text { reaction multifaceted for real time } \\
\text { application }\end{array}$ \\
\hline
\end{tabular}




\subsection{UUV modelling under actuated condition}

The thrusters are assembled in a design to provide the required drift to the vehicle. The open frame of the vehicle provides the best water flow, reducing the drag. Below actuated conditions is described as one having less control inputs than degree of freedom. Here some under actuated system, they require of actuation on definite guidelines or depth can be interpreted as constraints on the acceleration. For UUV, under actuated circumstance means when one or more of thrusters break down. The capability of to sustain a definite path or depth in its operation is address will be having two thrusters used for depth control. If one of thruster breaks down, the second thrusters will take over the control. The thrusters are having the $12 \mathrm{~V}$ to each.
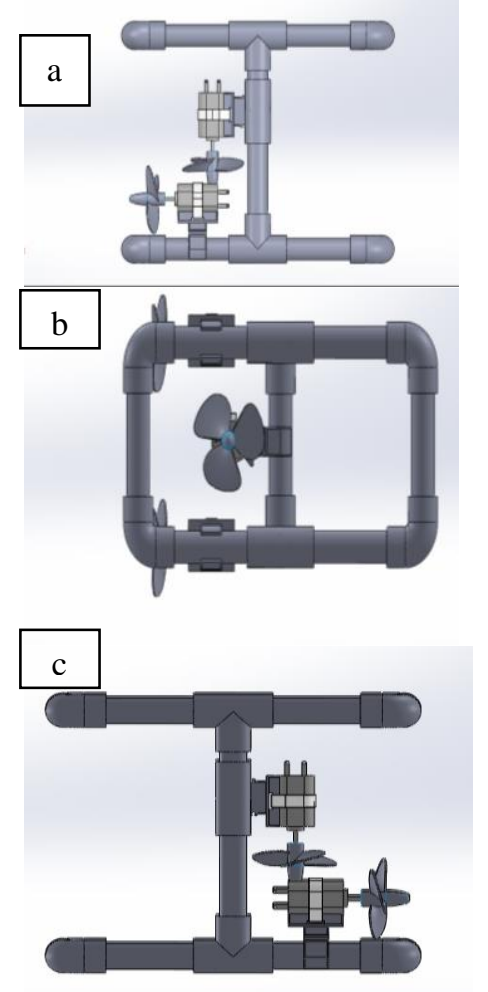
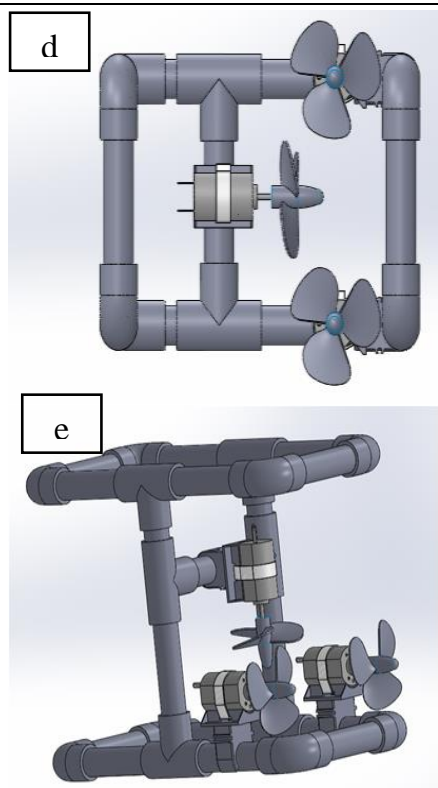

Fig.1. Thruster configuration (a) Top view of thruster (b) front view of thruster (c) Bottom view of thrusters (d) left view thruster (e) Right view of thrusters.

\subsection{Master and Slave Controller Tether}

The designed system is tethered through channel and remotely operates through an operative. Yoke material flanked by tether and wire design will be one of difficulty in solidity of system. Most of the systems entail a wire to convey the signal, video feedback, input voltage, and communication operator to the submerged vehicle. This will be attached stack to submerged Vehicle consignment, which are shown in figure 2 . The vehicle dimension, mass, working deepness, motors (thruster), subsystem, power, signal, power essential and pick and place is the constraint is must regard as in scheming. Here are two common types instead of wire; one is the Umbilical cable and the second is tether cable. Normal ROV used an electromechanical cable. 


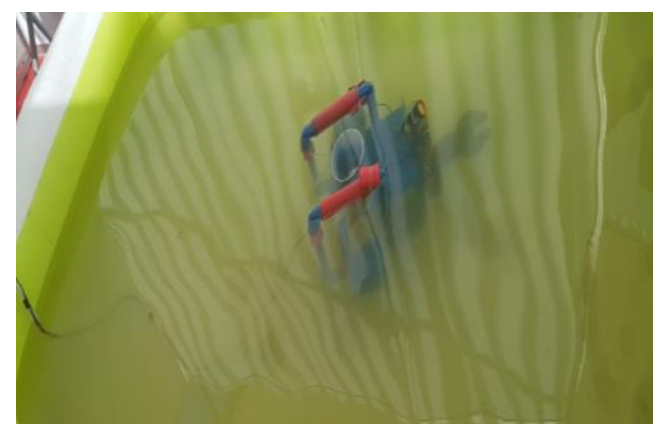

Fig .2. Working of tethered UUV in testing tank.

This wire, in fact, will be achieved the mechanics and solidity of UUV. So how we can decrease the dimension of wire for UUV application. During function in submerged is energized through the battery within the UUV, although DC brushless motor and live video feedback were energized through battery which is place at the surface. Examination, Exploring and video feedback is essential part of technology.

\subsection{Difficulties}

The unmanned underwater vehicle robotic system is characterized by the need for working in a difficult underwater environment. Any such robot need to handle various problems to function efficiently and effectively, such as waterproofing of the equipment, Buoyancy of the vehicle in the water, Communication and controlling of the vehicle in the water and Navigation in different directions.

We have overcome these problems by balancing the vehicle by adding dummy weight to the vehicle to minimize the buoyancy problem. To prevail overcome the problem of waterproof equipments, we made a water-proof box in which the circuitry of vehicle is placed to prevent it from water. We eliminated the problem of controlling servo motor by increasing its voltage. additional buttons and controls that are necessary for this project. Resembling the preceding editions of the ROV (remotely operated vehicle) control sketches, the PS2 controller is used for controlling equipment on the UUV such as the motors, motor direction, claw arm, and camera, as well as this prototype individual able to give the live video displayed on the topside station's LCD display.

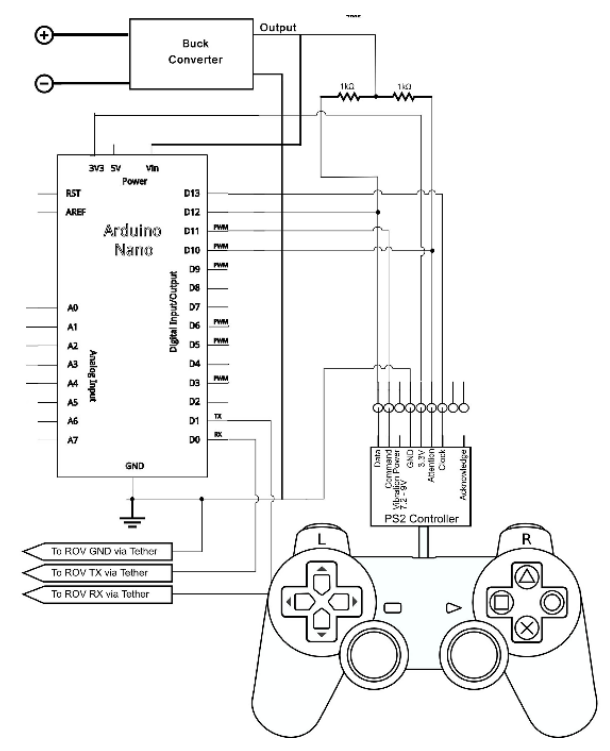

Fig. 3. Connection diagram of master controller.

Figure 3 shows the connection diagram of master controller.

In adding to the PS2 Controller, the topside station makes use of an Arduino Nano and a video LCD display. A buck converter is used to provide a $5 \mathrm{~V}$ to the Command and Attention pins of the PS2 controller and $12 \mathrm{~V}$ to LCD display, the command pin of PS2 is connected to pin D11 of the Arduino pin GND is connect to GND pin $3.3 \mathrm{v}$ to $3 \mathrm{v} 3$, clock pin is connect to D13 of the Arduino respectively.

\subsection{Controlling of UUV}

The Play station 2 controller is a pleasant economical control interface by 

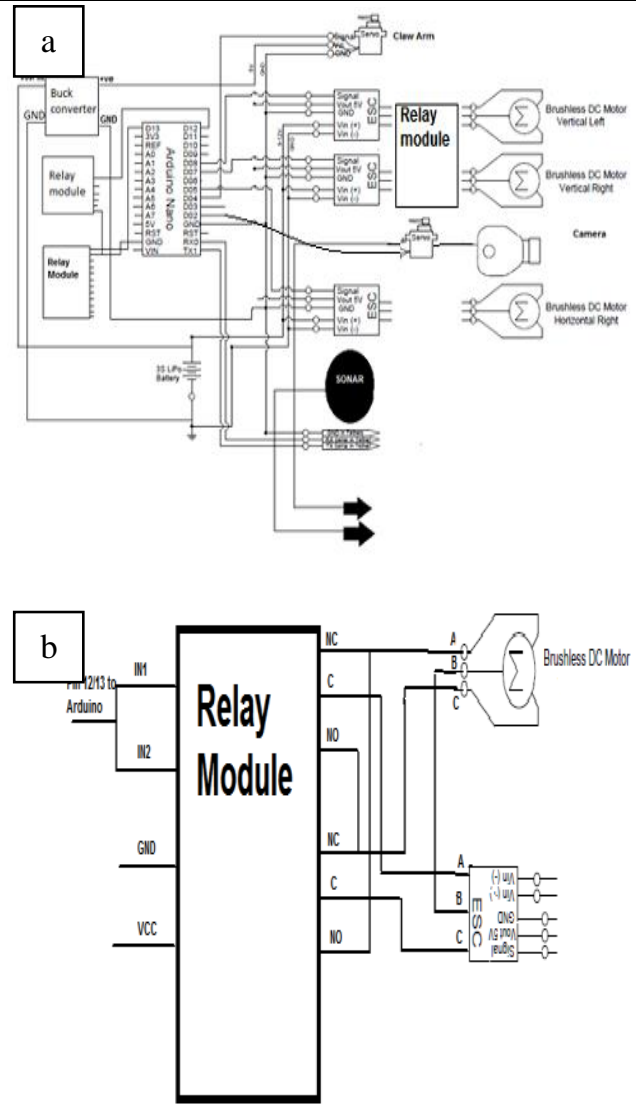

Fig.4.(a) Connection diagram of slave controller (b) connection diagram of thruster and relay module.

Fig. 4(a) shows the connection diagram of the salve controller and the connection of DC brushless motor also the connection of claw arm. The thrusters are connected to relay module, then the relay module is connected to the electronic speed controller (ESC). Fig. 4(b) shows the connection of thrusters and relay module.

The electronic circuit for the UUV is moderately uncomplicated because the majority of it is concerned with concerning fairly complicated equipment such as the speed controllers to the different pins on the Arduino. At this stage the board for the circuits is just paved collectively on Veroboard. formerly the circuit drawing as developed a appropriate circuit board will be developed.

\subsection{Working Principle}

Complete system operation is shown in system flow chart in Fig. 5 when user apply the instruction to the master controller through PS2 controller which are connected to the master controller and connection is shown in fig. 5, the system transmits the signal to the slave controller, with the salve controller the DC brushless motors are connected and servo motor of claw arm also connected. After receiving the signal from master controller, slave controller executes the function of the input signal which gives the feedback to the master controller. The input signal can be any which are defined already in programming that can perform the tasks like picking and placing the objects through claw arm, can also optimize the live feedback through camera and can also performs the task to move the vehicle in six directions.

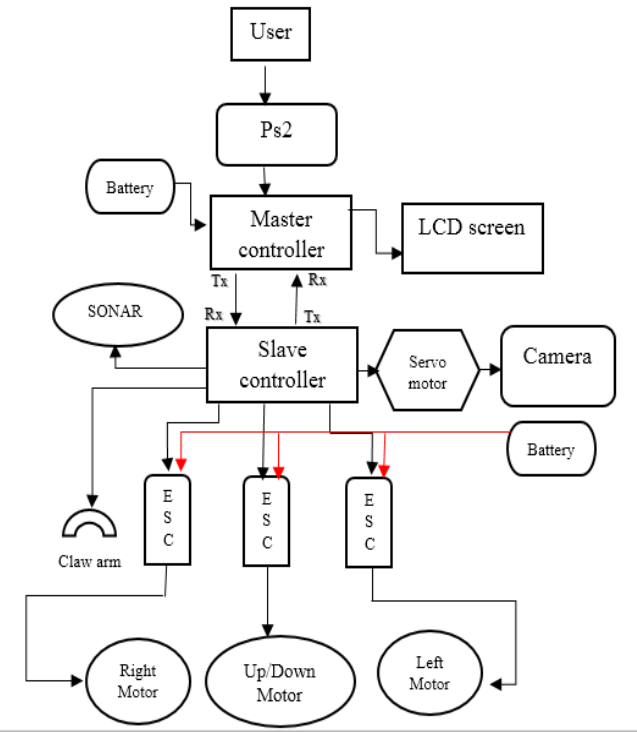

Fig. 5. Flow chart of UUV 


\section{Results and Discussion}

Throughout the working on UUV towards the completion of final prototype, it has been observed that the behavior of UUV was as per requirement. The designed system worked accurately as per desired depth. The movement of claw arm which worked in direction of $\mathrm{x}$-axis and camera, which moved in the direction of $y$-axis were accurate.it is used for underwater surveillance, inspection and for object recovery. It is the same as submarine but it has small size, low power consumption and low cost so it is more efficient. Initially the design system was not more efficient and functionally strong, which only works in four directions, there was no camera for live video feedback, no sonar for measuring its depth and no claw arm for picking and placing the objects. But in designed system is adding all these components which make it more efficient. The design vehicle is prototype model which performs all functions as described above. Fig. 6 shows the pulse width modulation (PWM) wave when we give the voltage to thrusters. The table II shows the characteristic of the prototype of Unmanned Underwater Vehicle (UUV).

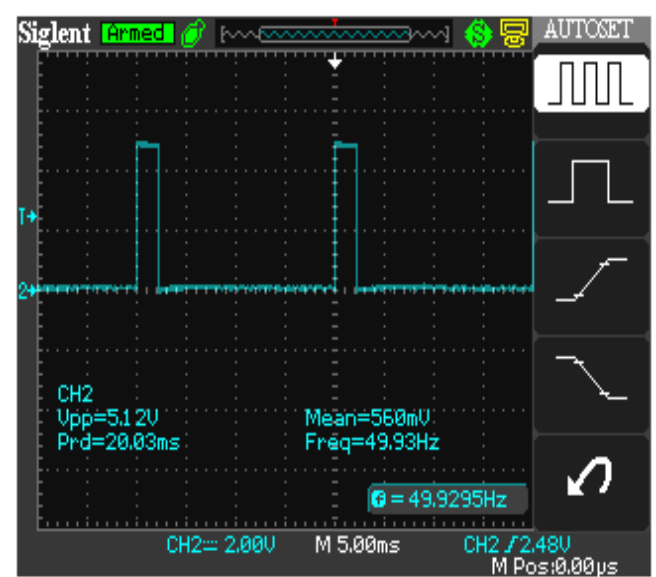

Fig.6. Shows the PWM of thrusters
TABLE II. Characteristics of UUV

\begin{tabular}{|l|l|}
\hline Specifications & Explanation \\
\hline Nominal speed & $0.8 \mathrm{~km} / \mathrm{h}$ \\
\hline Maximum speed & $1.5 \mathrm{~km} / \mathrm{h}$ \\
\hline Camera resolution & $12 \mathrm{Mp}, 1080 \mathrm{p}$ \\
\hline Degree of freedom & 3 \\
\hline Rated Voltage & $12 \mathrm{~V}$ \\
\hline Operating Depth & $5 \mathrm{~m}$ \\
\hline
\end{tabular}

\section{Conclusion}

The final designed Unmanned Underwater Vehicle has been demonstrated to be performing well and the developed UUV is remotely operated through a joystick controller and is able to perform inspections and surveillance of underwater environment efficiently. The UUV is well designed; it is waterproof, protecting the relevant equipment and sensors from water. The main tasks that it can perform are: navigation in six directions; up, down, left, right, forward and backward, imaging and visual monitoring of environment through camera and performing basic pick and place tasks using the claw arm. However, the final designed and developed has some limitations; for example, it can work up to 5 meters depth and communication is through cables only. Its manipulability capabilities are limited and require further sensors to effectively monitor underwater environment.

\section{Future Recommendation}

Above mentioned limitations can be overcome by adding autonomous control system. The arm complexity will be increased, which is currently limited by platform payload capacity and increase the depth range, pressure sensor to determine the operating depth, temperature sensor, leakage sensor and infrared sensors for thermal imaging. 


\section{REFERENCES}

[1] Paul G Fernandes, Pete Stevenson, and Andrew S Brierley, "Autonomous underwater vehicles: future platforms for fisheries acoustics," ICES Journal of Marine Science, Volume 60, Pages 684-691, 1 January 2003.

[2] Wallace M. Bessa, Max S. Dutra, and Edwin Kreuzer, "Depth control of remotely operated underwater vehicles using an adaptive fuzzy sliding mode controller", pp. 670-677, 26 November 2007.

[3] Ehsan Zakeri, Said Farahat and Seyed Alireza Moezi, "Robust sliding mode control of a mini unmanned underwater vehicle equipped with a new arrangement of water jet propulsions Simulation and experimental study" Applied Ocean Research, pp. 521-542, 2 June 2016.

[4] Salimzhan A. Gafurov and Evgeniy V. Klochkov, "Autonomous unmanned underwater vehicles development tendencies", pp. $141-148$.

[5] Othman M.N and Abdullah,S.S "Problem Identification for Underwater Remotely Operated Vehicle", pp. $554-560,2012$.

[6] Kumar Sandeep Jain, Sultan Mohammad, and Suyog Bora, "Autonomous Underwater Vehicle", Electronics and Communication, Department Vivekananda Institute of Technology (East) Jaipur, India, February-2015.
[7] Mallios Angelos, Ridao Pere, Marc Carreras and Hern'andez Emili, “ Navigating and Mapping with the SPARUS AUV in a Natural and Unstructured Underwater Environment", Campus de Montilivi, 17071, Girona, Spain, January 2012.

[8] Hardy Tim and Barlow Gavin, "Unmanned Underwater Vehicle deployment and retrieval considerations for submarines", INEC in Hamburg, Germany, April 2008.

[9] Norihide Wakita and Kiyoshi Hirokawa, "Development of Autonomous Underwater Vehicle (AUV) for Exploring Deep Sea Marine Mineral Resources", September 2010.

[10] Natural Resource consultants, "Remote Operated Vehicle Deep-water Crab Pot Removal Feasibility Study" Seattle, Washington 98119-1650, U.S.A, September 5, 2008

[11] L. Miller, D. Toal and E. Omerdic, "Low Cost Navigation for Unmanned Underwater Vehicles Using Line of Soundings" 2012, Pages 63-68.

[12] Antonio Fasano, Francesco Ferracuti and Alessandro Freddi, "A Virtual Thruster-Based Failure Tolerant Control Scheme for Underwater Vehicles" Universit a Campus Bio-Medico, Rome, Italy IFAC-PapersOnLine, Volume 48, Issue 16, 2015, Pages 146-151. 